\title{
DETERMINAÇÃO DE METAIS TRAÇO NO MATERIAL PARTICULADO EM SUSPENSÃO EM OURO PRETO, MINAS GERAIS
}

\author{
Lucas Carlúcio Magalhães \\ Centro Federal de Educação Tecnológica de Ouro Preto. Rua Pandiá Calógeras, s/nº, 35400-000 Ouro Preto - MG, Brasil \\ Hermínio Arias Nalini Junior \\ Departamento de Geologia, Escola de Minas, Universidade Federal de Ouro Preto, Campus Morro do Cruzeiro, 35400-000 Ouro \\ Preto - MG, Brasil \\ Aniel da Costa Lima e Mauricio Xavier Coutrim* \\ Departamento de Química, Instituto de Ciências Exatas e Biológicas, Universidade Federal de Ouro Preto, Campus Morro do \\ Cruzeiro, 35400-000 Ouro Preto - MG, Brasil
}

Recebido em 14/1/09; aceito em 23/10/09; publicado na web em 11/3/10

\begin{abstract}
DETERMINATION OF TRACE METALS IN SUPENDED PARTICULATE MATTER (SPM) IN OURO PRETO CITY, MINAS GERAIS. The determination of the airborne particulate matter (PTS) mass and trace metals concentrations were performed in three sites in Ouro Preto, MG, Brazil. It was evaluated 288 samples. The legal limit of annual geometric average $\left(60 \mu \mathrm{g} \mathrm{m}^{-3}\right)$ to PTS concentrations didn't exceed, but the legal limit for $24 \mathrm{~h}\left(240 \mu \mathrm{g} \mathrm{m}^{-3}\right)$ did in three measurements. Seven metals ( $\left.\mathrm{Al}, \mathrm{Cr}, \mathrm{Cu}, \mathrm{Fe}, \mathrm{Mn}, \mathrm{Ni}, \mathrm{Pb}\right)$ analysed by ICP-AES were quantified in 18 samples. Aluminum and iron showed the highest concentrations, indicating the contribution of the soil and of one aluminum plant. The high nickel concentration is probably due to vehicular emissions and industrial combustion processes.
\end{abstract}

Keywords: air quality; airborne particulate matter; trace metals.

\section{INTRODUÇÃO}

Tanto a saúde da população como as construções das cidades são diretamente afetadas pela poluição ambiental, de modo particular pela poluição atmosférica. Além dos gases presentes na atmosfera, destacam-se também as partículas suspensas no ar, constituídas de poeiras, fumaças e todo tipo de material sólido e líquido que se mantém suspenso na atmosfera por causa de seu pequeno tamanho, as quais têm um papel predominante nos processos regulados pela poluição atmosférica. ${ }^{1,2}$

As partículas em suspensão, dependendo do diâmetro médio, causam perturbação porque permanecem por longos períodos na atmosfera antes de serem removidas por mecanismos de depuração e por apresentarem espécies químicas tóxicas em sua superfície, como metais pesados e diversos compostos orgânicos. Essas partículas são constituídas de líquidos e sólidos e seu diâmetro médio varia de alguns nanômetros a cerca de $500 \mu \mathrm{m}$. As que realmente interferem na qualidade do ar de regiões urbanas se concentram em duas faixas de diâmetro médio: as partículas finas $(0,1$ a $2,5 \mu \mathrm{m}$; $\left.\mathrm{PM}_{2,5}\right)$ e as partículas grossas $(1,0$ a $100 \mu \mathrm{m})$. As partículas que possuem diâmetro médio menor do que $10 \mu \mathrm{m}\left(\mathrm{PM}_{10}\right)$ têm importância fundamental para o entendimento da contribuição da poluição atmosférica no ambiente, devido aos problemas relacionados à saúde humana provocados pelas mesmas. Essas partículas são respiráveis e, ao alcançar os pulmões, causam graves danos à saúde por conter componentes tóxicos ou mutagênicos em sua composição. Por outro lado, partículas com diâmetro médio superior a $100 \mu \mathrm{m}$ têm um tempo médio de permanência no ar muito pequeno e a ação dessas partículas no ambiente é limitada. ${ }^{3,4}$

As substâncias presentes no material particulado também podem danificar as rochas, causando prejuízos estéticos e criando condições ideais para catalisar processos de oxidação por meio de partículas de carbono e metais presentes na poeira, como manganês e ferro. ${ }^{5}$

*e-mail: mcoutrim@iceb.ufop.br
Muitos dos efeitos sobre a saúde humana são decorrentes da inalação de material particulado de diferentes tamanhos contendo metais em sua composição. Além do tamanho das partículas e as concentrações dos metais nas mesmas, suas características físico-químicas, solubilidade nos fluidos biológicos, o tempo total da exposição humana e o estado de saúde da população influenciam no efeito das partículas suspensas no ar na qualidade de vida numa região. ${ }^{6}$ Devido a isso, muitos estudos envolvendo a avaliação de metais presentes nas partículas suspensas no ar têm sido realizados em todo o mundo, como a avaliação de 17 metais presentes em partículas atmosféricas com diâmetro menor do que $10 \mu \mathrm{m}\left(\mathrm{PM}_{10}\right)$ em dois sítios na cidade de Palermo. ${ }^{7}$ Vários desses estudos têm sido desenvolvidos no Brasil. Pereira et al. ${ }^{8}$ avaliaram a presença de metais em poeiras atmosféricas em diversas frações de tamanho em três diferentes sítios na cidade de Salvador-BA e encontraram os metais $\mathrm{Fe}, \mathrm{Zn}$ e $\mathrm{Cu}$ em maior quantidade, cujas fontes foram atribuídas às atividades de mineração e veicular. A presença de metais no ar atmosférico de três importantes cidades do estado de São Paulo (São Paulo, Araraquara e Piracicaba) também foi determinada por Vasconcellos et al. ${ }^{9}$ que encontraram em maior concentração, além dos metais solúveis em água ( $\mathrm{Na}, \mathrm{K}, \mathrm{Na}$ e $\mathrm{Mg}$ ), Fe, Al, Ti, Cu e Zn, nessa ordem, e as fontes foram atribuídas a poeiras ressuspendidas do solo, queima de combustíveis de petróleo e indústrias metalúrgicas. Lara et $a l .{ }^{10}$ também avaliaram o ar atmosférico da cidade de Piracicaba com relação à presença de metais em partículas suspensas em duas faixas de diâmetros $\left(\mathrm{PM}_{2.5}\right.$ e $\left.\mathrm{PM}_{10}\right)$. Outros estudos com intuito de verificar a presença de metais em partículas presentes no ar atmosférico foram realizados em outras localidades do Brasil, como em Cabo Frio-RJ, ${ }^{11}$ onde dois sítios com contribuições predominantemente biogênicas foram avaliados, e em Capivari de Baixo-SC, ${ }^{12}$ onde foram avaliados sítios sob influência de emissões de usinas termoelétricas. Na cidade do Rio de Janeiro-RJ, estudos para avaliar a qualidade do ar com relação à presença de metais no material particulado foram realizados na Baixada Fluminense, ${ }^{13}$ região metropolitana onde as principais fontes de poluição são indústrias, emissões veiculares e naturais, e no distrito de Santa Cruz, ${ }^{14}$ uma região predominantemente industrial. 
A cidade de Ouro Preto, Patrimônio da Humanidade, com uma população em torno de 70 mil habitantes, mais de 55 mil pessoas vivendo na região urbana, possui uma topografia particular com várias formações montanhosas circundando todo o centro urbano da cidade..$^{15}$ Essas características geográficas, juntamente com um clima predominantemente frio e úmido, dificultam a dispersão de poluentes atmosféricos. Por outro lado, Ouro Preto abriga o maior acervo barroco do país, com muitas dessas obras de arte situadas a céu aberto e, devido aos efeitos dos poluentes atmosféricos sobre materiais expostos ao ar ambiente, ${ }^{16}$ o controle da qualidade do ar atmosférico nessa cidade é de suma importância. A qualidade do ar em Ouro Preto é afetada pela presença de indústrias de transformação, sendo que uma grande indústria de alumínio está localizada dentro do perímetro urbano, e pelo número crescente de veículos automotores, problema esse que é agravado pela malha viária urbana, com muitas ladeiras e ruas estreitas, fazendo com que a locomoção desses veículos aconteça em marchas reduzidas e com os motores em altas rotações (maior quantidade de combustível sendo queimado).

Amostras de partículas totais em suspensão (PTS) foram coletadas em três sítios em Ouro Preto durante 1 ano e as amostras foram avaliadas com relação à concentração, por gravimetria, e com relação à presença de diversos metais, por espectrometria de absorção atômica por plasma indutivamente acoplado (ICP-AES).

\section{PARTE EXPERIMENTAL}

\section{Área de estudo}

A cidade de Ouro Preto, MG, tem uma temperatura média anual de $17,4^{\circ} \mathrm{C}$. A precipitação média anual é de $2018 \mathrm{~mm}$, com distribuição bastante irregular e concentrada no período compreendido entre os meses de outubro a março. O clima é classificado como tropical de altitude e os ventos predominantes são de sudeste para noroeste. As amostras foram coletadas em três sítios: o primeiro (PILAR) situado no bairro Pilar (20²3`13,0”'S e 43³0’30,0”W, $1090 \mathrm{~m}$ de altitude), o segundo (FEBEM) no prédio da antiga Fundação Estadual do Bem-Estar do Menor de Minas Gerais, atualmente ocupado pela Secretaria de Saúde da Prefeitura Municipal de Ouro Preto (20²2'54,8'S e 43³1'19,4'W, 1122 m de altitude) e o terceiro (EM) no prédio da Escola de Minas da Universidade Federal de Ouro Preto (UFOP) na Praça Tiradentes, região central da cidade (20²3'02,5'S e 43³0'14,3”W, $1145 \mathrm{~m}$ de altitude). Os sítios PILAR e EM estão localizados no centro histórico da cidade de Ouro Preto, enquanto que o sítio FEBEM está localizado no limite oeste da cidade, região sujeita a uma menor influência de poluentes atmosféricos provenientes de fontes antropogênicas locais (Figura $1 \mathrm{~S}$, material suplementar). Duas fontes fixas importantes são uma fábrica de alumínio localizada próxima ao centro histórico de Ouro Preto $\left(20^{\circ} 23^{\prime} 56,1^{\prime \prime} \mathrm{S}\right.$ e $43^{\circ} 31^{\prime} 08,2^{\prime \prime} \mathrm{W}, 1100 \mathrm{~m}$ de altitude) e uma fábrica de ferro-ligas a cerca de $6 \mathrm{~km}$ a sudoeste do centro histórico (20²5'05,8'S e 4333'06,9'W, 1315 m de altitude).

\section{Procedimento de amostragem}

As partículas totais em suspensão (PTS) foram coletadas em todos os sítios a cada 6 dias no período de maio de 2002 a maio de 2003 , utilizando-se amostradores de grandes volumes $(\mathrm{Hi}-\mathrm{Vol})$ da marca Energética, mod. AGV, totalizando 288 amostras. ${ }^{17}$ Utilizaram-se filtros de fibra de vidro, da marca Energética, mod. E055, com 203 x $254 \mathrm{~mm}$. Depois de mantidas em dessecador contendo sílica gel por $24 \mathrm{~h}$, as massas dos filtros foram medidas em balança analítica marca Mettler, mod. AB 204 e, em seguida, os filtros foram colocados no amostrador para a retenção do PTS, mantendo-se a aspiração do ar por um período contínuo de $24 \mathrm{~h}$ e, em seguida, foram mantidos novamente em dessecador por mais $24 \mathrm{~h}$ antes da determinação da massa dos mesmos em balança analítica. A concentração de PTS, expressa em $\mu \mathrm{g} \mathrm{m}^{-3}$, foi determinada relacionando-se a massa de PTS coletada no filtro e o volume de ar amostrado.

Nas 18 amostras com maiores concentrações de PTS coletadas em cada sítio foram determinadas as concentrações dos metais Al, $\mathrm{Fe}, \mathrm{Zn}, \mathrm{Cr}, \mathrm{Cu}, \mathrm{Mn}, \mathrm{Ni}, \mathrm{Pb}, \mathrm{As}, \mathrm{Be}, \mathrm{Cd}$, Co e Mo. Uma área correspondente a $3 \%$ da área amostrada no filtro foi retirada e submetida inicialmente à extração com $4 \mathrm{~mL}$ de solução ácida $\left(55 \mathrm{~mL} \mathrm{HNO}_{3}\right.$ conc e $165 \mathrm{~mL} \mathrm{HCl}$ conc para $1 \mathrm{~L}$ em água) por $1 \mathrm{~h}$ e, após adição de $2 \mathrm{~mL}$ de água ultrapura (MilliQ ${ }^{\circledR}$ Plus), em micro-ondas, marca CEM Corporation, modelo MDS 2000, a $640 \mathrm{~W}$ e 40 psi por $10 \mathrm{~min}$, depois a 80 psi por $5 \mathrm{~min}$ e, finalmente, 100 psi por $5 \mathrm{~min}$. Um filtro não amostrado foi utilizado para a determinação do branco. Após avolumar o material extraído com água Milli Q a $25 \mathrm{~mL}$, os metais foram determinados na solução final por ICP-AES nos respectivos comprimentos de onda de maior sensibilidade do equipamento da marca Spectro modelo Ciros/CCD. Para a verificação da recuperação do método de extração utilizou-se um material de referência, NIST 1640 , e os valores encontrados estiveram entre 90 e $110 \%$.

\section{RESULTADOS E DISCUSSÃO}

\section{Partículas totais em suspensão (PTS)}

A estratégia de escolha dos sítios e da confecção do calendário de amostragem buscou uma abrangência que melhor representasse a área estudada, isto é, o centro urbano de Ouro Preto. Embora durante o dia a direção preferencial dos ventos na região estudada seja de sudeste (Figura $1 \mathrm{~S}$, material suplementar), à noite ela é de sudoeste. As localizações dos sítios de amostragens privilegiaram as regiões da cidade que à noite recebem grande parte das emissões da fábrica de alumínio localizada na região urbana. Os resultados de concentrações de PTS no ar atmosférico de Ouro Preto encontrados são apresentados na Tabela 1, através da média geométrica, dos menores e maiores valores por sítio de amostragem, juntamente com os limites preconizados pelo padrão de qualidade do ar (PQAr) ${ }^{18} \mathrm{e}$ com resultados de avaliações similares realizadas em outras cidades do Brasil. ${ }^{19,20}$ Com relação a todas amostras dos 3 sítios avaliados $(\mathrm{n}=288), 3$ resultados ultrapassaram a concentração limite de PTS para amostragens de $24 \mathrm{~h}$ do padrão primário $\left(240 \mu \mathrm{g} \mathrm{m}^{-3}\right)$ e 16 resultados estiveram acima do padrão secundário $\left(150 \mu \mathrm{g} \mathrm{m}^{-3}\right)$. Os resultados obtidos na estação do Pilar com relação à concentração máxima e ao número de vezes de ultrapassagem do padrão primário são piores do que aqueles encontrados no bairro Pinheiros, em São Paulo. Porém, são melhores do que aqueles obtidos na Vila Parisi, em Cubatão e se assemelham aos obtidos no bairro Sider Ville, em Volta Redonda. A concentração média geométrica anual limite para o padrão secundário $\left(60 \mu \mathrm{g} / \mathrm{m}^{3}\right)$ não foi ultrapassada em nenhum dos três locais de amostragem em Ouro Preto, o que não aconteceu nos outros sítios comparados na Tabela 1.

Na estação do Pilar, o padrão primário legal para períodos de 24 $\mathrm{h}$ foi excedido três vezes, não estando, portanto, em conformidade com a legislação nacional vigente. ${ }^{18}$ Pela legislação esse padrão não deve ser excedido mais de uma vez ao longo de 1 ano. O padrão secundário para períodos de $24 \mathrm{~h}$ foi excedido 12 vezes, nesse local de amostragem, ao longo do período de estudo. No entanto, a legislação prevê que somente os padrões primários são aplicáveis na avaliação da qualidade do ar, enquanto não for definida a classificação do território nacional de acordo com o uso pretendido. ${ }^{18}$

Na Figura 1 são apresentados todos os resultados de concentração encontrados nas amostras coletadas, em ordem cronológica 
Tabela 1. Concentrações de PTS em amostras coletadas em 3 sítios em Ouro Preto no período de maio de 2002 a maio de 2003 comparadas com limites do PQAr e resultados de avaliação em São Paulo, SP, em 2002, e em Volta Redonda, RJ, em 2007

\begin{tabular}{|c|c|c|c|c|c|c|}
\hline \multirow{2}{*}{ Local } & \multirow{2}{*}{$\mathrm{n}$} & \multirow{2}{*}{$\begin{array}{l}\text { Conc. mínima } \\
\quad\left(\mu \mathrm{g} \mathrm{m}^{-3}\right)\end{array}$} & \multirow{2}{*}{$\begin{array}{l}\text { Conc. máxima } \\
\qquad\left(\mu \mathrm{g} \mathrm{m}^{-3}\right)\end{array}$} & \multicolumn{2}{|c|}{$\mathrm{N}^{\circ}$ de vezes ultrapassado } & \multirow{2}{*}{$\begin{array}{l}\text { Conc. média geom. } \\
\qquad\left(\mu \mathrm{g} \mathrm{m}^{-3}\right)\end{array}$} \\
\hline & & & & Pad. Prim. & Pad. Sec. & \\
\hline Pad. Prim..$^{18}$ & & & 240 & & & 80 \\
\hline Pad. Sec. ${ }^{18}$ & & & 150 & & & 60 \\
\hline PILAR & 96 & 9,0 & 285,0 & 3 & 12 & 56,7 \\
\hline EM & 96 & 8,0 & 194,0 & 0 & 4 & 43,7 \\
\hline FEBEM & 96 & 5,2 & 102,1 & 0 & 0 & 31,7 \\
\hline Pinheiros, São Paulo, $\mathrm{SP}^{19}$ & 56 & ND & 150 & 0 & ND & 63 \\
\hline V. Parisi, Cubatão, $\mathrm{SP}^{19}$ & 58 & ND & 486 & 22 & ND & 192 \\
\hline Sider Ville, Volta Redonda, $\mathrm{RJ}^{20}$ & 60 & ND & 295 & 5 & ND & 95 \\
\hline
\end{tabular}

ND - Não disponível

para os três locais de amostragem. Também são apresentadas nessa figura as concentrações limites para PTS estabelecidas pelo PQAr para os padrões primário e secundário com o objetivo de facilitar a visualização dos resultados das amostras que ultrapassaram esses limites. ${ }^{18} \mathrm{O}$ período compreendido entre os meses de setembro de 2002 e março de 2003 foi responsável por $89,7 \%$ da precipitação total. O mês de janeiro de 2003 registrou o maior índice de precipitação pluviométrica (Figura 1).

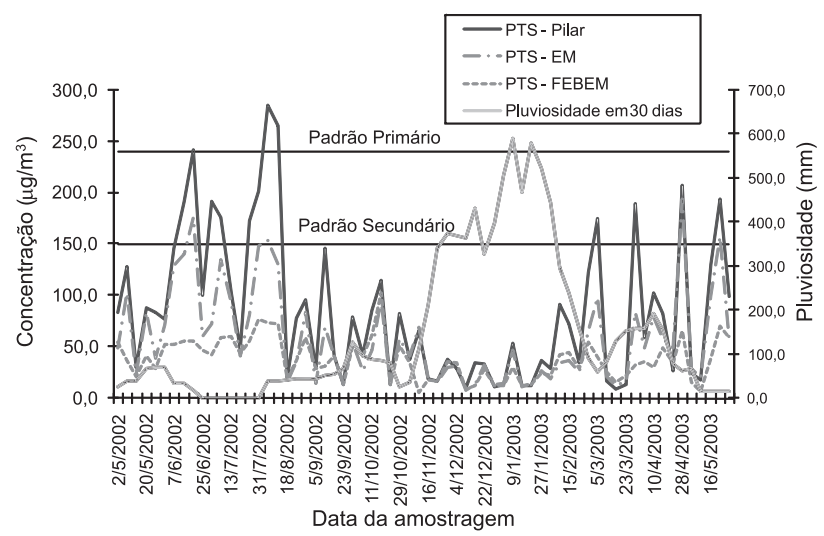

Figura 1. Concentrações de PTS nas estações de amostragem, comparadas com o PQAr e a pluviosidade nos 30 dias anteriores

As maiores concentrações para o PTS na estação do Pilar foram obtidas nos meses de junho a agosto de 2002 e março a maio de 2003. Esse período corresponde ao inverno e ao outono no hemisfério sul, justamente os períodos de maior estiagem nessa parte do globo terrestre. Em Ouro Preto isso não foi diferente, conforme constatado na Figura 1. No período de estiagem, a falta de chuva provoca um tempo maior de permanência do PTS na atmosfera. Por outro lado, as mais baixas concentrações de PTS na estação do Pilar foram obtidas nos meses de primavera e verão, pois as precipitações favorecem a depuração atmosférica, promovendo a remoção das partículas suspensas por deposição úmida. ${ }^{21}$ Este comportamento sazonal foi também observado na cidade argentina de La Plata, ${ }^{22}$ assim como em estudos realizados em outras cidades brasileiras, como São Carlos. ${ }^{23} \mathrm{Em}$ cidades do hemisfério norte, como Cartagena - Espanha e Copenhagen - Dinamarca, nos meses de outono e inverno também foi observada maior concentração de PTS na atmosfera em estudos similares. ${ }^{24}$

Nas estações da FEBEM e da Escola de Minas, as concentrações de PTS não excederam uma única vez o padrão primário de $240 \mu \mathrm{g} \mathrm{m}^{-3}$ para períodos de $24 \mathrm{~h}$. Na estação da FEBEM nem mesmo o padrão secundário de $150 \mu \mathrm{g} \mathrm{m}^{-3}$ foi ultrapassado. ${ }^{18} \mathrm{Na}$ estação da Escola de Minas, este limite foi ultrapassado 4 vezes no período estudado.

Com relação aos episódios críticos, definidos pela legislação como aqueles caracterizados quando as concentrações de PTS alcançam os valores de $375,625 \mathrm{e} 875 \mu \mathrm{g} \mathrm{m}^{-3}$, levando ao estabelecimento dos níveis de atenção, alerta e emergência para a qualidade do ar, respectivamente, não foram verificados em nenhuma das amostras obtidas. Entretanto, na estação do Pilar o padrão primário de $240 \mu \mathrm{g}$ $\mathrm{m}^{-3}$ para o período de $24 \mathrm{~h}$ foi ultrapassado em três medições (Tabela 1). Dentre as três localizações avaliadas, a do bairro do Pilar foi a que apresentou os piores resultados. Nas estações da FEBEM e da Escola de Minas o padrão primário não foi ultrapassado uma única vez (Tabela 1). Assis et al. ${ }^{25}$, em estudo semelhante desenvolvido no período de 2000 a 2002, também obtiveram resultados mais altos nesta estação. As maiores concentrações de PTS foram encontradas na estação Pilar, $330 \mu \mathrm{g} \mathrm{m}^{-3}$ nos anos de 2000 e 2001 e $285 \mu \mathrm{g} \mathrm{m}^{-3}$ no ano de 2002, enquanto que nas estações Escola de Minas e FEBEM esses valores foram iguais a 264, 256 e $176 \mu \mathrm{g} \mathrm{m}^{-3} \mathrm{e} 124,120$ e 102 $\mu \mathrm{g} \mathrm{m}{ }^{-3}$, respectivamente..$^{25}$

\section{Metais no PTS}

Em 18 amostras de cada um dos três sítios avaliados foram determinadas as concentrações dos seguintes metais: Al, Fe, Zn, $\mathrm{Cr}, \mathrm{Cu}, \mathrm{Mn}, \mathrm{Ni}, \mathrm{Pb}, \mathrm{As}, \mathrm{Be}, \mathrm{Cd}$, Co e Mo, sendo que os 5 últimos apresentaram valores de concentração abaixo do limite de quantificação. O silício, porque estava presente no material do filtro, e os metais cujos íons são solúveis em água, não foram determinados nesse estudo ( $\mathrm{Na}, \mathrm{K}, \mathrm{Mg}$ e $\mathrm{Ca}$ ). O zinco foi determinado nas amostras, mas somente após o término do trabalho foi constatado que os filtros utilizados nas amostragens apresentavam alto teor de zinco em suas constituições. Dessa forma, somente 7 metais foram quantificados nas amostras coletadas nos três sítios avaliados. Alumínio e ferro apresentaram os maiores valores de concentração em todas as amostras, uma vez que o solo na região contém grande quantidade de minério de ferro, fazendo com que este metal esteja presente na poeira ressuspensa no local. As presenças de uma fábrica de ferroligas e de outra de alumínio próximas aos locais de amostragens contribuem fortemente para as altas concentrações desses metais no PTS. Além disso, há uma extensa área sem cobertura vegetal formada por material laterítico/bauxítico, rico em $\mathrm{Al}_{2} \mathrm{O}_{3}$, situada a sul-sudeste do sítio FEBEM, que é menos impactado pelo tráfego urbano e as fábricas. Porém, a direção preferencial de ventos de sudeste faz com que a contribuição da ressuspensão de poeira do solo nesse sítio seja significativa.

Avaliando-se as concentrações dos metais em cada sítio de amostragem (Tabela 2) verifica-se que os elementos $\mathrm{Al}, \mathrm{Cr}, \mathrm{Ni}$ e $\mathrm{Pb}$ apresentaram concentrações mais elevadas na estação do Pilar, evidenciando ser este sítio o mais impactado ambientalmente por material particulado e metais associados, no período estudado. As concentrações de Fe e Mn mais elevadas ocorreram na estação da 
FEBEM. Este ponto, por estar situado no limite da cidade, está mais sujeito à contribuição de material particulado originado de rochas intemperizadas, especialmente o itabirito rico em ferro e contendo manganês associado. Apesar disso, a concentração média de manganês encontrada nesse sítio é quase 7 vezes menor que aquela encontra no distrito de Santa Cruz, Rio de Janeiro-RJ, local fortemente impactado por atividades industriais. ${ }^{14}$ Resultados de avaliações em outros estudos têm demonstrado que o sistema de freio veicular, principalmente de veículos mais pesados, como ônibus e caminhões, libera cobre para a atmosfera. ${ }^{8,26,27}$ As concentrações mais elevadas de cobre foram registradas na Escola de Minas e podem estar relacionadas ao intenso tráfego veicular nas ruas próximas ao local de amostragem..$^{28}$ Resultados semelhantes foram obtidos em avaliação do PTS e partículas inaláveis $\left(\mathrm{PM}_{10}\right)$ realizada no bairro Belmonte, em Volta Redonda, Rio de Janeiro (Tabela 3). ${ }^{29}$

Tabela 2. Concentrações de metais, em ng m ${ }^{-3}$, em 18 amostras de PTS coletadas em 3 sítios em Ouro Preto no período de maio de 2002 a maio de 2003

\begin{tabular}{lcccccc}
\hline \multirow{2}{*}{ Sítio } & \multicolumn{2}{c}{ PILAR } & \multicolumn{2}{c}{ EM } & \multicolumn{2}{c}{ FEBEM } \\
& Média & Intervalo & Média & Intervalo & Média & Intervalo \\
\hline $\mathrm{Al}^{*}$ & 32,6 & $2,6-54,3$ & 26,1 & $16,7-35,1$ & 23,1 & $18,1-28,0$ \\
$\mathrm{Cr}$ & 16,3 & $12,0-23,2$ & 15,5 & $12,5-18,7$ & 15,7 & $12,7-20,0$ \\
$\mathrm{Cu}$ & 165,0 & $27,0-349,0$ & 209,9 & $55,0-411,0$ & 159,4 & $75,0-289,0$ \\
$\mathrm{Fe}^{*}$ & 2,8 & $0,5-5,6$ & 3,0 & $1,2-5,8$ & 3,1 & $0,8-6,1$ \\
$\mathrm{Mn}$ & 113,5 & $17,7-211,3$ & 96,1 & $35,7-240,6$ & 177,6 & $28,7-550,0$ \\
$\mathrm{Ni}$ & 47,3 & $6,9-115,0$ & 32,5 & $7,6-80,9$ & 13,9 & $7,9-38,1$ \\
$\mathrm{~Pb}$ & 41,7 & $13,1-109,4$ & 19,2 & $13,0-45,7$ & 15,6 & $13,4-25,4$ \\
\hline
\end{tabular}

*Concentrações expressas em $\mu \mathrm{g} \mathrm{m}^{-3}$.

Os valores mais altos de concentração de níquel encontrados nos três sítios comparados a outros estudos (Tabela 3) podem estar relacionados à presença desse metal em diversos processos de combustão, tais como as veiculares e industriais. ${ }^{30}$ As emissões da queima de óleo combustível e de coque do processo Bayer de produção de alumínio pela fábrica de Ouro Preto, um processo eletroquímico de fusão da alumina com criolita a cerca de $1000{ }^{\circ} \mathrm{C}$ utilizando coque como anodo, possivelmente contribuíram para as maiores concentrações de níquel registradas, sendo que o sítio com maiores concentrações desse metal (PILAR) é o que sofre mais influência da fábrica.

Vale ressaltar que as concentrações dos metais pesados não ultrapassaram os limites estabelecidos pela agência de controle ambiental norte-americana, EPA, ${ }^{31}$ e pela Organização Mundial de Saúde, WHO, ${ }^{32}$ conforme pode ser verificado na Tabela 3. Deve-se ressaltar que esses limites de concentração foram estabelecidos para a fração inalável do material particulado $\left(\mathrm{PM}_{10}\right)$ e que aproximadamente $80 \%$ das partículas em suspensão na atmosfera correspondem a esta fração, sendo válida, portanto, a comparação. ${ }^{33}$

\section{CONCLUSÕES}

Os resultados de concentração diária de 24 h de PTS encontrados ultrapassaram o padrão primário legal de $240 \mu \mathrm{g} \mathrm{m}^{-3}$ pelo menos três vezes no período do estudo. As concentrações de PTS apresentaram variação sazonal. As precipitações pluviométricas influíram nos valores registrados, pois os níveis mais elevados ocorreram nos meses de outono e inverno, de baixa precipitação, e as menores concentrações ocorreram nos meses de primavera e verão, de maior frequência de chuvas.

Com relação aos metais presentes no PTS, as maiores concentrações foram encontradas para alumínio e ferro, sendo o primeiro muito provavelmente originado das emissões de uma fábrica de alumínio e o segundo tem origem nas emissões de uma fábrica de ferro-ligas, além da ressuspensão do solo. As concentrações de $\mathrm{Cr}$, Mn, Ni e Pb
Tabela 3. Concentrações de metais, em ng $\mathrm{m}^{-3}$, em Ouro Preto, MG, e em outras localidades com limites de exposição da EPA e WHO

\begin{tabular}{|c|c|c|c|c|c|c|c|}
\hline Local & $\mathrm{A} 1^{*}$ & $\mathrm{Cr}$ & $\mathrm{Cu}$ & $\mathrm{Fe}^{*}$ & $\mathrm{Mn}$ & $\mathrm{Ni}$ & $\mathrm{Pb}$ \\
\hline PILAR & 32,6 & 16,3 & 165,0 & 2,8 & 113,5 & 47,3 & 41,7 \\
\hline EM & 26,1 & 15,5 & 209,9 & 3,0 & 96,1 & 32,5 & 19,2 \\
\hline FEBEM & 23,1 & 15,7 & 159,4 & 3,1 & 177,6 & 13,9 & 15,6 \\
\hline Nova Iguaçu, $\mathrm{RJ}^{13}$ & 1,001 & 3,1 & 42,0 & 1,7969 & 54,5 & 1,2 & 11,9 \\
\hline Mesquita, $\mathrm{RJ}^{13}$ & 1,4129 & 3,8 & 49,3 & 1,9743 & 49,4 & 0,4 & 21,7 \\
\hline Santa Cruz, $\mathrm{RJ}^{14}$ & 5,774 & 421 & 335 & 38,903 & 1216 & 0,5 & 101,0 \\
\hline Tito Scalo, Itália ${ }^{21}$ & - & 13,0 & 58,0 & 0,5 & 27,0 & 5,0 & 60,0 \\
\hline Coimbatore, Índia ${ }^{26}$ & - & 14,2 & 388,6 & - & - & 31,4 & 143,5 \\
\hline $\begin{array}{l}\text { Volta Redonda, } \\
\mathrm{RJ}^{29}\end{array}$ & - & - & 160,0 & 4,0 & 200,0 & 16,0 & 224,0 \\
\hline Limite $\mathrm{EPA}^{31}$ & - & 100,0 & - & - & 500,0 & 200,0 & 1500,0 \\
\hline Limite $\mathrm{WHO}^{32}$ & - & 1100,0 & - & - & 150,0 & - & 500,0 \\
\hline
\end{tabular}

*Concentrações expressas em $\mu \mathrm{g} \mathrm{m} \mathrm{m}^{-3}$; ( - ) não disponível.

presentes no PTS foram inferiores, em todos os sítios amostrados, aos limites estabelecidos por agências reguladoras para esses metais. As maiores concentrações de $\mathrm{Cu}$ ocorreram nas amostras coletadas na Escola de Minas, ponto de amostragem mais impactado por tráfego veicular, indicando ser essa a principal fonte para esse metal.

\section{MATERIAL SUPLEMENTAR}

A Figura 1S contendo o mapa com a localização dos sítios de amostragem do PTS em Ouro Preto, MG, está disponível gratuitamente em http://quimicanova.sbq.org.br, na forma de arquivo PDF.

\section{AGRADECIMENTOS}

Ao Programa de Pós-graduação em Engenharia Ambiental da UFOP. À J.C. Pereira pela ajuda nas análises por ICP-AES.

\section{REFERÊNCIAS}

1. WHO - World Health Organization; Health aspects of air pollution with particulate matter, ozone and nitrogen dioxide, WHO: Genebra, 2003.

2. Baird, C.; Química Ambiental, 2ª ed., Bookman: Porto Alegre, 2002.

3. Seinfeld, J.; Pandis, S. N.; Atmospheric Chemistry and Physics: from air pollution to climate change, Wiley: New Jersey, 1998.

4. WHO - World Health Organization. Guidelines for air quality, WHO: Genebra, 2000.

5. Camuffo, D.; Grieken, R. V.; Busse, H-J.; Sturaro, G.; Valentino, A.; Bernardi, A.; Blades, N.; Shooter, D.; Gysels, K.; Deutsch, F.; Wieser, M.; Kim, O.; Ulrych, U.; Atmos. Environ. 2001, 35, S127.

6. Voutsa, D.; Samara, C.; Atmos. Environ. 2002, 36, 3583.

7. Dongarrà, G.; Manno, E.; Varrica, D.; Vultaggio, M.; Atmos. Environ. 2007, 41, 7977

8. Pereira, P. A. P.; Lopes, W. A.; Carvalho, L. S.; Rocha, G. O.; Bahia, N. C.; Loyola, J.; Quiterio, S. L.; Escaleira, V.; Arbilla, G.; de Andrade, J. B.; Atmos. Environ. 2007, 41, 7837.

9. Vasconcellos, P. C.; Balasubramanian, R.; Bruns, R. E.; Sanchez-Ccoyllo, O.; Andrade, M. F.; Flues, M.; Water, Air, Soil Pollut. 2007, 186, 63.

10. Lara, L. L.; Artaxo, P.; Martinelli, L. A.; Camargo, P. B.; Victoria, R. L.; Ferraz, E.S.B.; Atmos. Environ. 2005, 39, 4627.

11. Sella, S. M.; Neves, A. F.; Moreira, J. C.; Silva-Filho, E. V.; Atmos. Environ. 2006, 40, 6181.

12. Godoy, M. L. D. P.; Godoy, J. M.; Artaxo, P.; Atmos. Environ. 2005, 39, 5307.

13. Quiterio, S. L.; Sousa, C. R. S.; Arbilla, G.; Escaleira, V.; Atmos. Environ. 2005, 39, 3503. 
14. Quiterio, S. L.; Sousa, C. R. S.; Arbilla, G.; Escaleira, V.; Atmos. Environ. 2004, 38, 321.

15. http://www.ibge.gov.br/cidadesat/, acessada em Fevereiro 2007.

16. Kucera,V.; Fitz, S.; Water, Air, Soil Pollut. 1995, 85, 153.

17. ABNT - Associação Brasileira de Normas Técnicas; Determinação da concentração total pelo método do amostrador de grandes volumes, NBR-9547, ABNT: Rio de Janeiro, 1997.

18. CONAMA - Conselho Nacional de Meio Ambiente; Resolução 03, CONAMA: Brasília, 1990.

19. CETESB - Cia. Tecnologia e Saneamento Ambiental; Relatório de Qualidade do Ar no Estado de São Paulo - 2002, CETESB: São Paulo, 2003.

20. FEEMA - Fundação Estadual de Engenharia do Meio Ambiente; Relatório Anual de Qualidade do Ar - 2007, disponível em www.inea. rj.gov.br/fma/relatório_2007.pdf., acessada em Julho 2009.

21. Ragosta, M. C. R.; D'emilio, M.; Macchiato, M. M.; Atmos. Environ. 2002, 36, 3071.

22. Bilos, C.; Colombo, J. C.; Skorupka, C. N.; Presa, M. J. R.; Environ. Pollut. 2001, 1, 111.

23. Celli, C. E.; Marques, K. A.; Teixeira, D.; Bachiega, E.; Machado, A. P.; Carvalho, W. V.; Aguiar, M. L.; Coury, J. R.; Eng. Sanit. Ambient. 2003, 8,6 .
24. Moreno-Grau, S.; Pérez-Tornell, A.; Bayo, J.; Moreno, J.; Angosto, J. M.; Moreno-Clavel. J.; Atmos. Environ. 2000, 34, 5161.

25. Assis, C. M.; Silveira, I. L.; Anizelli, R. C. M.; Anais do III Congresso Interamericano de Qualidade do Ar, Canoas, Brasil, 2003.

26. Mohanraj, R.; Azeez, P. A.; Priscilla, T.; Arch. Environ. Contam. Toxicol. 2004, 47, 162.

27. Artaxo, P.; Castanho, A.; Atmos. Environ. 2001, 35, 4889.

28. Steven, H. C.; Mulawa, P. A.; Balli, J.; Donase, C.; Weibel, A.; Sagebiel, J. C.; Environ. Sci. Technol. 1997, 31, 3405.

29. Gioda, A.; Sales, J. A.; Cavalcanti, P. M. S.; Maia, M. F.; Maia, L. F. P. G.; Aquino Neto, F. R.; J. Braz. Chem. Soc. 2004, 15, 856.

30. Dongarrà, G.; Manno, E.; Varrica, D.; Vultaggio, M.; Atmos. Environ. 2007, 41, 7977.

31. EPA - US Environmental Protection Agency, disponível em www.atsdr. cdc.gov., acessada em Dezembro 2008.

32. WHO - World Health Organization; Air Quality Guidelines for Europe, WHO: Copenhagen, 2000.

33. FEPAM - Fundação de Proteção Ambiental do Rio Grande do Sul; Avaliação da poluição atmosférica, FEPAM: Porto Alegre, 1998. 


\section{DETERMINAÇÃO DE METAIS TRAÇO NO MATERIAL PARTICULADO EM SUSPENSÃO EM OURO PRETO, MINAS GERAIS}

Lucas Carlúcio Magalhães

Centro Federal de Educação Tecnológica de Ouro Preto. Rua Pandiá Calógeras, s/nº, 35400-000 Ouro Preto - MG, Brasil

Hermínio Arias Nalini Junior

Departamento de Geologia, Escola de Minas, Universidade Federal de Ouro Preto, Campus Morro do Cruzeiro, 35400-000 Ouro Preto - MG, Brasil

Aniel da Costa Lima e Mauricio Xavier Coutrim*

Departamento de Química, Instituto de Ciências Exatas e Biológicas, Universidade Federal de Ouro Preto, Campus Morro do Cruzeiro, 35400-000 Ouro Preto - MG, Brasil

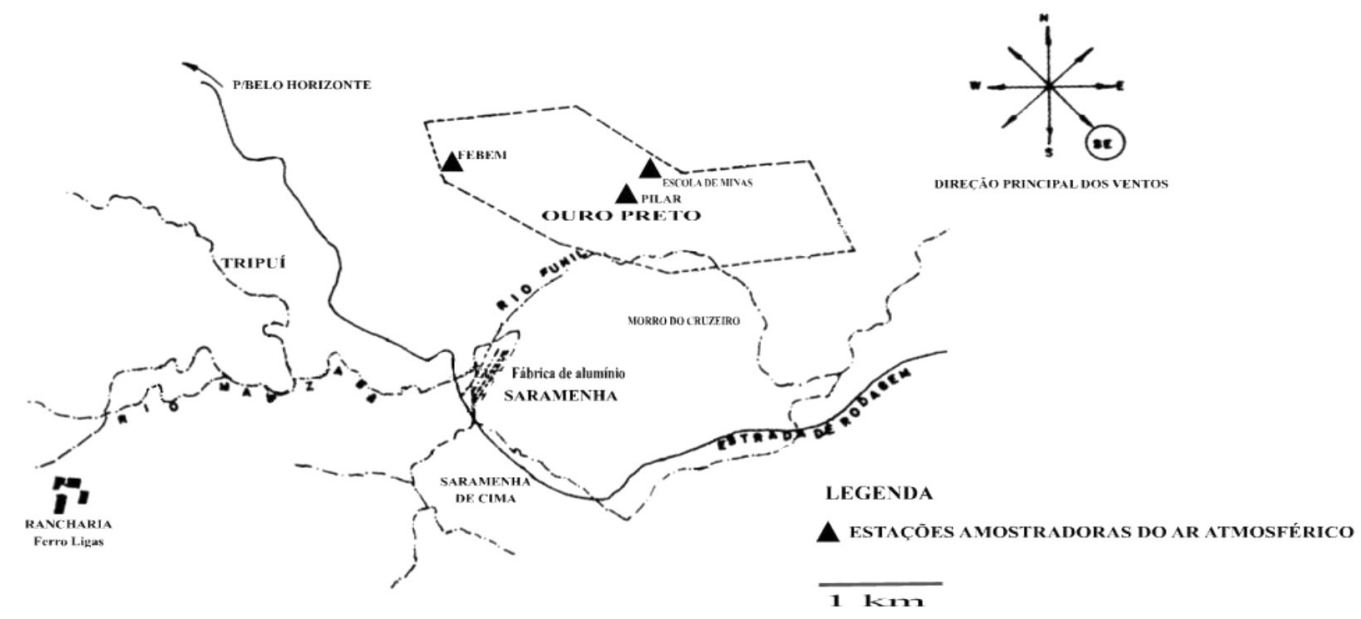

Figura 1S. Localização dos sítios de amostragem das partículas totais em suspensão (PTS) coletadas do ar atmosférico em Ouro Preto, MG

*e-mail: mcoutrim@iceb.ufop.br 